\title{
Articles
}

\section{Hyperglycaemic siblings of Type II (non-insulin-dependent) diabetic patients have increased PAI-1, central obesity and insulin resistance compared with their paired normoglycaemic sibling}

\author{
O. M. Herlihy ${ }^{1}$, B. A. Barrow ${ }^{1}$, P. J. Grant ${ }^{2}$, J. C. Levy ${ }^{1}$ \\ ${ }^{1}$ Diabetes Research Laboratories, Radcliffe Infirmary, Oxford, United Kingdom \\ 2 Unit of Molecular Vascular Medicine, Leeds School of Medicine, Leeds, UK
}

\section{Abstract}

Aims/hypothesis. First-degree relatives of Type II (non-insulin-dependent) diabetic patients in cross-sectional studies have increased insulin resistance, associated cardiovascular risk factors and abnormalities of fibrinolysis and coagulation. To minimise betweenfamily genetic and environmental confounders, we investigated within-family relationships between early hyperglycaemia and risk factors.

Methods. Thirteen age and gender matched sibling pairs of Type II (non-insulin-dependent) diabetic patients, one hyperglycaemic, one normoglycaemic (fasting plasma glucose at screening $6.0-7.7 \mathrm{mmol} \cdot \mathrm{l}^{-1}$ and $<6.0 \mathrm{mmol} \cdot \mathrm{1}^{-1}$, respectively) were assessed for plasminogen activator inhibitor antigen (PAI-1), tissue plasminogen activator antigen (t-PA), fibrinogen, Factor VII and Factor VIII/von Willebrand factor antigen. Fasting lipid profiles, blood pressure and HOMA insulin sensitivity $(\% \mathrm{~S})$ were also measured in siblings and in matched subjects without family history of diabetes. Results. Hyperglycaemic and normoglycaemic siblings ( 7 female, 6 male) were aged, mean (SD) 56.8
(8.7) and 55.8 (8.4) years. Hyperglycaemic siblings had increased PAI-1 antigen, geometric mean (i.q.r.): $26.3(15.1-45.6)$ vs $11.1(2.1-23.3) \mathrm{ng} / \mathrm{ml}, p=0.0002$, similar t-PA antigen, mean (SD) 9.5 (4.3) vs 7.4 (2.5) $\mathrm{ng} / \mathrm{ml}, p=0.2$ and fibrinogen $2.2(0.3)$ vs $2.3(0.6) \mathrm{g} / \mathrm{l}$, $p=0.5$, and reduced $\% \mathrm{~S} 66.3$ (30.5) vs 82.9 (25), $p=0.04$. PAI-1 correlated negatively with $\% \mathrm{~S}$ $(r=-0.55, p=0.005)$. No significant differences were found in blood pressure or fasting lipids.

Conclusion/interpretation. A minor increase in plasma glucose in non-diabetic sibling pairs of Type II (noninsulin-dependent) diabetic patients was associated with reduced insulin sensitivity, increased central adiposity and a doubling of PAI-1 antigen concentration, suggesting impaired fibrinolysis. It is possible that this could contribute to increased cardiovascular risk in these subjects. [Diabetologia (2002) 45:635-641]

Keywords Hyperglycaemia, Type II diabetes, blood coagulation, fibrinolysis, PAI-1, tissue plasminogen activator, insulin sensitivity, central obesity, sibling, lipids.
Received: 14 May 2001 / Revised: 24 December 2001

Published online: 26 April 2002

(C) Springer-Verlag 2002

Corresponding author: Dr. J. C. Levy, Diabetes Research Laboratories, Oxford Centre for Diabetes, Endocrinology and Metabolism, Radcliffe Infirmary, Oxford OX2 6HE, UK, e-mail: jonathan.levy@drl.ox.ac.uk

Abbreviations: PAI-1, Plasminogen activator inhibitor-1; t-PA, plasma tissue plasminogen activator; LDL vWF, von Willebrand factor: low density lipoprotein; FVII, factor VII; FVIII, factor VIII; FXIII, factor XIII; VLDL, very low density lipoprotein; HDL, high density lipoprotein; log, logarithm to the base 10
Type II (non-insulin-dependent) diabetic patients have a two to threefold increase in morbidity and mortality from cardiovascular disease $[1,2]$. This excess morbidity and mortality is not explained fully by the presence of recognised risk factors such as hypertension, dyslipidaemia or obesity [3], though standard measures of these traits are relatively crude. Abnormalities of haemostasis predict the occurrence of cardiovascular disease in the general population $[4,5,6$, 7] and an increased thrombotic tendency has also been demonstrated in Type II diabetic patients, who have higher concentrations of both coagulation and anti-fibrinolytic factors (including fibrinogen, factor 
VII (FVII), von Willebrand factor (vWF) in the presence of microalbuminuria, plasminogen activator inhibitor 1 (PAI-1) and plasma tissue plasminogen activator (t-PA) $[8,9,10])$. The combination of recognised risk factors and abnormalities of haemostasis could account for the increased cardiovascular mortality of the disease.

Non-diabetic first-degree relatives of Type II diabetic subjects have an increased prevalence of cardiovascular disease and a clustering of cardiovascular risk factors (obesity, hypertension, dyslipidaemia, impaired glucose tolerance) associated with insulin resistance $[9,10,11,12,13,14]$. The syndrome of insulin resistance has been shown to be associated with abnormalities of haemostasis, particularly suppression of fibrinolysis $[15,16]$. First-degree relatives of patients with Type II (non-insulin-dependent) diabetes have also been shown to have higher concentrations of FVII-C, fibrinogen [17] and PAI-1 [18].

We investigated the relationship between metabolic and haemostatic abnormalities and mild hyperglycaemia in siblings of Type II diabetic patients. Diabetic sibships, however, exhibit considerable heterogeneity with respect to insulin resistance and associated metabolic variables, due to a combination of environmental and genetic factors. As this heterogeneity could mask underlying relationships or give rise to spurious associations between these variables we specifically studied differences between age and sex matched non-diabetic sibling pairs selected for discordance for fasting plasma glucose concentration.

\section{Subjects and methods}

Thirteen pairs of siblings from different families, matched for sex and age (less than 5 years difference between the siblings) but discordant for fasting plasma glucose (FPG), were chosen from a population-based collection of sibships of patients with Type II diabetes consisting of a proband plus at least two other siblings living within 50 miles of Oxford, the Diabetes in Families Collection (DIF). Siblings were categorised as normoglycaemic or hyperglycaemic on the basis of the screening FPG of less than $6.0 \mathrm{mmol}^{-1}$ or FPG of more than $6.0 \mathrm{mmol}^{-1}$ but less than $7.8 \mathrm{mmol} \cdot \mathrm{l}^{-1}$. A further 13 non-diabetic control subjects were individually matched to the normoglycaemic siblings for sex, age, BMI and fasting glucose from 460 subjects aged 35-70 years with no first-degree relative with diabetes, who comprised a subgroup of a general population sample studied in parallel with the DIF population. Normoglycaemic and hyperglycaemic siblings, but not control subjects, were studied on a further day for metabolic and haemostatic variables. All subjects gave informed consent and the study was approved by the Central Oxford Research and Ethics Committee.

Subjects attended the department following a 10-h overnight fast (water only allowed). After a $30 \mathrm{~min}$ rest period, blood samples were drawn from the antecubital vein using a 20 gauge cannula, without a tourniquet. Blood was taken into $0.9 \%$ citrate $(\mathrm{pH} 8.8)$ on ice in the ratio of 9 parts blood to
1 part citrate for assay of t-PA and PAI-1antigen. These samples were centrifuged at $2000 \mathrm{~g}$ at $4^{\circ} \mathrm{C}$ for $30 \mathrm{~min}$. Blood for clotting factors (FVII, FVIIIC, FXa, FXIIA, FXIIIB subunit and FXIIIB subunit) and von Willebrand Factor antigen (vWFag) was collected into $0.9 \%$ citrate and centrifuged at $2000 \mathrm{~g}$ at room temperature for $30 \mathrm{~min}$. Insulin, proinsulin and C-peptide were assayed in lithium heparin plasma, glucose in fluoride oxalate plasma and plasma lipid analysis in EDTA plasma. Insulin sensitivity $(\% \mathrm{~S})$ and beta-cell function $(\% \mathrm{~B})$ were calculated using the HOMA model with fasting glucose-insulin and glucose-C-peptide pairs, respectively [19, 20].

Blood pressure was measured with the subject sitting. Systolic and diastolic recordings were taken from the mean of three readings. Waist circumference was measured at the midpoint between the costal margin and the superior iliac spine. BMI was calculated from weight in kilos divided by the height in metres squared.

Assays. Fasting plasma glucose measurements was determined by the Glucose Oxidase Membrane method (YSI, Hampshire, UK). Glucose and Oxygen from Hydrogen peroxide which is oxidised at the anode. Analysis is performed using the YSI 2300 Glucose Analyser. Sample collection was into fluoride oxalate tubes which were stored on ice until separation. Samples were assayed within $24 \mathrm{~h}$. Plasma immunoreactive insulin was determined by double antibody radioimmunoassay (RIA) with Sepharose attached to the second antibody for separation by decanting (PhRIA100, Pharmacia Ltd, Milton Keynes, Bucks, UK). There was $100 \%$ cross-reaction to intact proinsulin and cross-reaction with all split proinsulins in this RIA [21]. Plasma C-peptide was assayed by a wide range radio-immunoassay using ${ }^{125}$ I human $\mathrm{C}$-peptide with guinea pig anti-human C-peptide ( $<4 \%$ cross reactivity to human proinsulin) and polyethylene glycol (PEG) precipitation (Linco $\mathrm{Hu}-$ man C-peptide RIA kit, Biogenesis, Poole, UK). Analysis was performed using a liquid Handling System, IDS Star 700 (Kemble Instrument, Sussex, UK). Plasma proinsulin was determined by a two-site sandwich ELISA, using murine monoclonal antibodies PEP-001 (reactive to human C-peptide) and HUI-001 (reactive to the beta chain of human insulin). The assay has $100 \%$ cross reactivity with intact proinsulin (PI) and $74 \%$ cross reactivity with $32-33$ split PI, $65 \%$ with des (30, 31) split PI and no cross reactivity with insulin or C-peptide. Total, LDL and HDL cholesterol and triglycerides were measured using an enzymatic colorimetric methods (Boehringer Mannheim Cholesterol CHOD-PAP method) on the Cobas FARA centrifugal analyser (Roche Diagnostic Systems, Lewes, East Sussex, UK). HDL cholesterol was measured following precipitation of LDL and VLDL with sodium heparin and manganese II chloride and LDL cholesterol was calculated after measurement of VLDL cholesterol by precipitation of LDL and HDL cholesterol with sodium dodecyl sulphate [21]. Plasma PAI-1 antigen and tPA antigen were measured by enzyme-linked immunosorbent assay (Imulyse, Biopool, Umea, Sweden). Interassay and intra-assay CV's (9.7\% and 6.0\%) for PAI-1 and $(9.5 \%$ and $7.0 \%)$ for tPA respectively. Fibrinogen was measured by the method of Clauss CV's $(3.5 \%$ and $2.0 \%$ ) [22]. Von Willebrand Factor was measured by ELISA, (Dako, Ely, Cambridgeshire, UK) CV's (7.8\% and $4.4 \%$ ). Factor's VII and VIII were measured by an ACL3000 (Instrumentation Laboratories, Milan, Italy). Factor XII was assayed using an ELISA technique (Shield activated factor kit, (Shield Diagnostics, Dundee, UK). Factor XIII Activity was measured with a one-stage microtitre assay using fibrinogen and 5-(biotinamide) pentylamin as substrates CV's $7.3 \%$ and $3.9 \%$ [23]. 
Table 1. Characteristics of the sibling pairs

\begin{tabular}{|c|c|c|c|c|c|}
\hline \multirow[b]{2}{*}{$n$} & \multirow{2}{*}{$\begin{array}{l}\begin{array}{l}\text { Normoglycaemic } \\
\text { siblings }\end{array} \\
13\end{array}$} & \multirow{2}{*}{$\begin{array}{l}\text { Hyperglycaemic } \\
\text { siblings }\end{array}$} & \multirow{2}{*}{$\begin{array}{l}p \text { between } \\
\text { sibs } \\
\text { (pairwise) }\end{array}$} & \multirow{2}{*}{$\begin{array}{l}\begin{array}{l}\text { Control } \\
\text { population }\end{array} \\
13\end{array}$} & \multirow{2}{*}{$\begin{array}{l}p \text { between normo- } \\
\text { glycaemic siblings } \\
\text { and control subjects } \\
\text { (groupwise) }\end{array}$} \\
\hline & & & & & \\
\hline men/women & $6: 7$ & $6: 7$ & (matched) & $6: 7$ & 1.0 \\
\hline FPG $\left(\mathrm{mmol} \cdot \mathrm{l}^{-1}\right)$ & $5.3(0.29)$ & $6.3(0.73)$ & (selected) & $5.3(0.3)$ & 0.87 \\
\hline Smoker : non-smoker & $4: 9$ & $5: 8$ & 0.68 & $1: 11$ & 0.32 \\
\hline $\mathrm{BMI}\left(\mathrm{kg} \cdot \mathrm{m}^{-2}\right)$ & $26.3 \pm 3.3$ & $27.8 \pm 3.6$ & 0.20 & $26.1 \pm 3.1$ & 0.60 \\
\hline Waist circumference $(\mathrm{cm})$ & $89 \pm 14$ & $94 \pm 11$ & 0.01 & $88 \pm 14$ & 0.78 \\
\hline Systolic BP (mm Hg) & $137 \pm 23$ & $137 \pm 24$ & 0.97 & $137 \pm 28$ & 0.93 \\
\hline Diastolic BP (mm Hg) & $89 \pm 10$ & $86 \pm 19$ & 0.61 & $83 \pm 11$ & 0.09 \\
\hline Total cholesterol ${ }^{\mathrm{a}}\left(\mathrm{mmol} \cdot \mathrm{l}^{-1}\right)$ & $5.5(4.5-6.5)$ & $5.0(4.3-6)$ & 0.10 & $5.8(4.9-6.8)$ & 0.20 \\
\hline LDL cholesterola $\left(\mathrm{mmol} \cdot \mathrm{l}^{-1}\right)$ & $3.5(2.5-4.9)$ & $3.3(2.6-4.2)$ & 0.53 & $3.6(2.8-4.6)$ & 0.75 \\
\hline
\end{tabular}

a Values are geometric means (1 SD range) for total cholesterol, LDL, HDL, triglycerides

Table 2. Comparison of beta-cell function and insulin sensitivity in sibling pairs

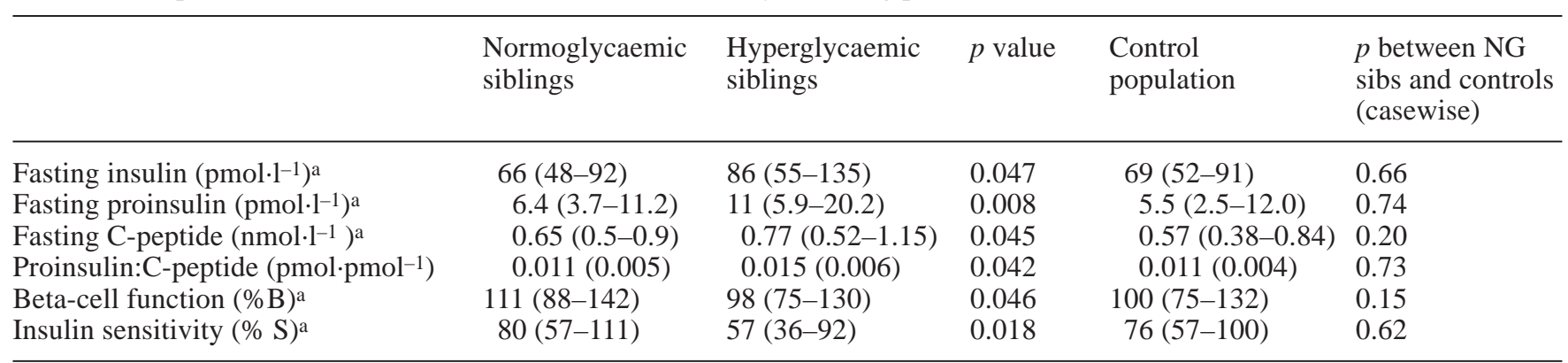

a Values are expressed as geometric means (1 SD range). Insulin sensitivity and beta-cell function were calculated using the HOMA model [32, 33]

Calculations and statistics. Values were checked for skewdness (skewness statistic >2SE). This was corrected by logarithmic transformation for total-, LDL-, HDL-cholesterol, triglycerides, fasting insulin, proinsulin, C-peptide, beta-cell function, insulin sensitivity and PAI-1 antigen. Statistical analysis was performed on transformed values. Summary statistics are reported as mean (1 SD) or geometric mean (1 SD range), where appropriate. As siblings were family-matched and agematched, comparisons between the normoglycaemic and hyperglycaemic groups were performed using paired $t$ tests. Pearson correlations were performed using transformed variables. Stepwise multiple linear regression was performed with probability thresholds for entry and removal of 0.05 and 0.10 , respectively). Individual matching of control subjects was done by choosing the member of the whole control group having the minimum Mahalanobis distance from each NG sibling for sex, age, BMI and FPG. Statistical analyses were performed using SPSS for Windows 9.0 (SPSS, Chicago, Ill., USA).

\section{Results}

The characteristics of the siblings are shown in Table 1 . One HG sibling was taking a statin and another was taking a beta-blocker. No NG sibling was taking any prescribed medication. In a self-completed questionnaire, $9 \mathrm{NG}$ and $10 \mathrm{HG}$ sibs described themselves as 'sedentary', $3 \mathrm{NG}$ and $1 \mathrm{HG}$, 'moderately active' (on their feet for at least $4 \mathrm{~h}$ a day) and $0 \mathrm{NG}$ and $3 \mathrm{HG}$, 'active' (on their feet most of the day). HG and NG did not differ significantly with respect to age, weight or BMI (mean and 95\%-CI of differences: 1.1 $(-1.3-3.4)$ years, $1.6(-0.7-3.9) \mathrm{kg} \cdot \mathrm{m}^{-2}$, for age and BMI, respectively). HS were more centrally obese and had a lower insulin sensitivity (HOMA \%S), a lower pancreatic beta-cell function (HOMA \%B) and a higher proinsulin-to-C-peptide ratio compared with NS (Table 2). However, there was no significant difference in either systolic or diastolic blood pressure or plasma lipid profiles (mean and 95\%-CI of differences: $0(-14-15)$ and $-3(-13-7) \mathrm{mm} \mathrm{Hg},-0.4$ $(-0.8-0.0),-0.2(-0.6-0.2),-0.12(-0.29-0.08)$ and $0.2(-0.4-0.7) \mathrm{mmol} \cdot \mathrm{l}^{-1}$ for systolic and diastolic blood pressures and total-, LDL-, HDL-cholesterol and triglycerides, respectively. There were no significant differences between NG siblings and matched 
Table 3. Comparison of PAI-1, t-PA and clotting factor levels in sibling pairs

\begin{tabular}{|c|c|c|c|}
\hline & Normoglycaemic siblings & Hyperglycaemic siblings & $p$ value \\
\hline PAI-1antigen $\left(\mathrm{ng} \cdot \mathrm{ml}^{-1}\right)^{\mathrm{a}}$ & $11.1(5.3-23.3)$ & $26.2(15.1-45.6)$ & 0.0002 \\
\hline $\mathrm{t}-\mathrm{PA}$ antigen $\left(\mathrm{ng} \cdot \mathrm{ml}^{-1}\right)$ & $7.4(5.2-10.5)$ & $8.9(5.9-13.5)$ & 0.22 \\
\hline Factor VIIc (units $\cdot \mathrm{ml}^{-1}$ ) & $1.3(1.0-1.7)$ & $1.4(1.0-1.8)$ & 0.39 \\
\hline Factor VIIIc (unit·ml-1) & $1.4(0.4)$ & $1.4(0.4)$ & 0.61 \\
\hline von Willebrand factor (unit·ml-1) & $1.2(0.5)$ & $1.1(0.4)$ & 0.58 \\
\hline
\end{tabular}

a Values are geometric mean (1 SD range), t-PA other clotting factors are expressed as means (SD)

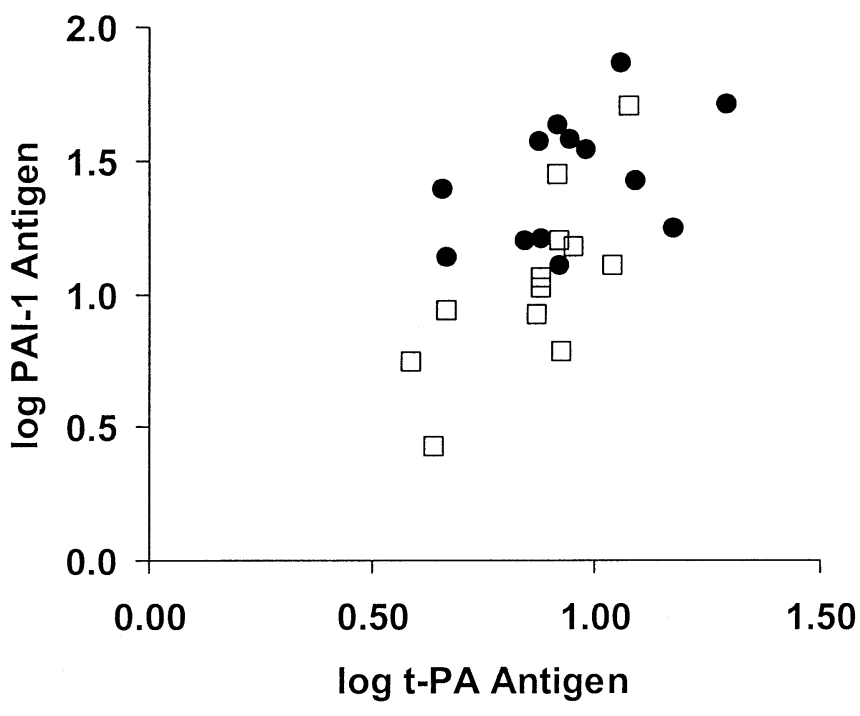

Fig. 1. Scattergram of PAI-1 antigen concentrations and t-PA concentration in normoglycaemic $(\square)$ and hyperglycaemic sibling pairs

controls who did not have a first-degree relative with diabetes (Table 1).

Concentrations of PAI-1antigen were significantly higher in the HG. No significant differences were found in t-PA antigen, FVII, FVIIIc, FXIIa, FXIII, fibrinogen or vWFag (Table 3).

In the combined group of hyperglycaemic and normoglycaemic sibs, a strong correlation was seen between log PAI-1 and log t-PA (Pearson $R=0.61$, $p<0.001)$, though PAI- 1 was additionally raised in the HG siblings ( $p=0.005$, Fig. 1$)$. Both PAI- 1 and t-PA were positively correlated with waist circumference and negatively correlated with insulin sensitivity and HDL-cholesterol but PAI-1 was additionally correlated with BMI and t-PA with triglycerides (Table 4). Within each group, PAI-1 correlated with waist circumference ( $p=0.022$ and 0.008 in the NG and $\mathrm{HG}$ groups respectively) but with no other variables (Fig. 2A), whereas t-PA correlated with waist circumference $(p=0.014$, Fig. 2B) and HDL-cholesterol $(p=0.021)$ only in the NG siblings. In a stepwise re-
Table 4. Pearson correlation coefficients ( $\mathrm{R}$ ( $p$ value)) with PAI-1 antigen and t-PA concentration in combined hypoglycaemic and normoglycaemic sibling groups $(n=26)$

\begin{tabular}{lll}
\hline & PAI-1 Ag & t-PA \\
\hline log t-PA & 0.61 & \\
& $(0.001)$ & \\
Age (years) & 0.27 & 0.39 \\
& $(0.19)$ & $(0.054)$ \\
BMI (kg.m-2) & 0.57 & 0.27 \\
& $(0.002)$ & $(0.19)$ \\
Waist (cm) & 0.65 & 0.58 \\
& $(<0.001)$ & $(0.003)$ \\
Systolic BP (mm Hg) & 0.30 & 0.18 \\
& $(0.14)$ & $(0.40)$ \\
Diastolic BP (mm Hg) & 0.21 & 0.23 \\
& $(0.29)$ & $(0.27)$ \\
Log plasma glucose (mmol.1-1) & 0.38 & 0.31 \\
& $(0.065)$ & $(0.13)$ \\
Triglycerides & 0.26 & 0.45 \\
& $(0.21)$ & $(0.028)$ \\
Log plasma HDL-cholesterol (mmol.1-1) & -0.40 & -0.49 \\
& $(0.046)$ & $(0.015)$ \\
Log plasma NEFA (umol.1-1) & -0.08 & 0.08 \\
Log plasma proinsulin (pmol·1 ${ }^{-1}$ ) & $(0.73)$ & $(0.71)$ \\
Log HOMA \%S & 0.55 & 0.51 \\
& $(0.004)$ & $(0.010)$ \\
& -0.61 & -0.42 \\
& $(0.001)$ & $(0.038)$ \\
\hline
\end{tabular}

gression of log PAI-1 against glycaemic status (NG vs HG) and anthropomorphic and Metabolic Syndrome variables (age, waist circumference, waist-hip ratio, BMI, triglycerides, log HDL cholesterol, NEFA, log proinsulin and log HOMA \%S), the only significant independent factors were glycaemic status and waist circumference, $(p<0.001$ and 0.007 , respectively, adjusted $R^{2}=0.57$ ). In a similar stepwise regression of log t-TPA against the same factors only waist circumference was significant $(p=0.003$, $\left.R^{2}=0.32\right)$. 


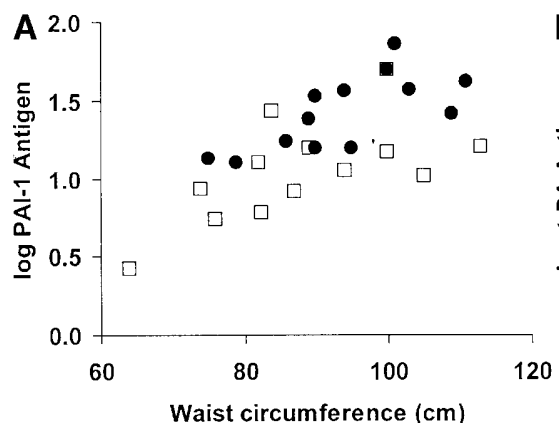

Fig. 2A, B. Scattergram of PAI-1 antigen (A) and t-PA concentration (B) with waist circumference in normoglycaemic ( $\square$ ) and hyperglycaemic $(\square)$ sibling pairs

\section{Discussion}

These data show that in non-diabetic sibling pairs of Type II diabetic patients discordant for fasting glycaemia, hyperglycaemic siblings (fasting plasma glucose 6.0-7.7 mmol. $\cdot^{-1}$ ) had increased waist circumference, decreased insulin sensitivity and an increased fasting proinsulin-to-C-peptide ratio, with increased concentrations of PAI-1 antigen, though no other significant differences in features of the Metabolic Syndrome or coagulation variables.

The study aimed to focus on the features that accompany minor elevations in glycaemia in non-diabetic siblings of Type II diabetic patients. In cross-sectional studies, non-diabetic first degree relatives of Type II diabetic patients have been shown to have increased obesity, insulin resistance and an increased prevalence of other cardiovascular risk factors associated with the Metabolic Syndrome compared with unrelated controls $[11,12,14,24]$. However, Type II diabetes is a heterogeneous condition and this is reflected in between-family differences and within-family similarities in insulin resistance and other features of the Metabolic Syndrome, including obesity, hypertension and dyslipidaemia. This has been described in Pima Indians [25], and also in the UK Diabetes in Families Study (DIF) from which the sibling pairs in this study were drawn. Such stratification could give rise to spurious associations in cross-sectional studies. For example, in the DIF collection, the prevalence of hyperglycaemia was greater in sibships with decreased insulin sensitivity (personal communication). A simple cross-sectional recruitment of hyperglycaemic siblings would be disproportionately drawn from families with increased insulin resistance, with the potential for generating misleading associations. This selection bias would be reduced by a within-family study design as has been used here.

This study has therefore adopted a within-family approach in the comparison between hyperglycaemic

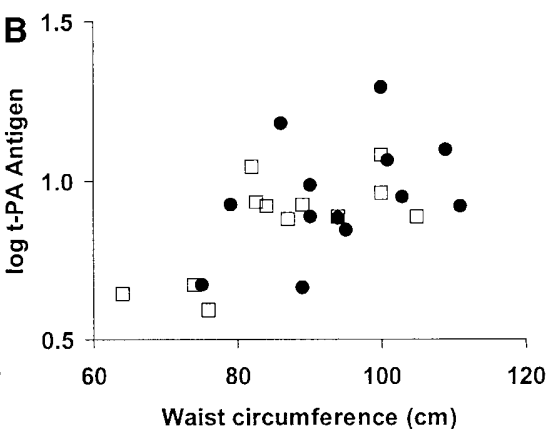

and normoglycaemic siblings to reduce potential confounding genetic and environmental factors between families. Sibling pairs were chosen to match for sex and age, but to be discordant with respect to glycaemic control. Hyperglycaemia was defined on the basis of fasting plasma glucose to include subjects lying above the normal range, but below the 1985 World Health Organisation (WHO) threshold for the diagnosis of diabetes to reduce the chance of secondary metabolic effects of moderate or marked hyperglycaemia. However, only two of the 13 hyperglycaemic siblings had values above the current ADA and WHO thresholds for diabetes.

The hyperglycaemic subjects were more insulin resistant than their normoglycaemic siblings, and in keeping with this, fasting insulin and proinsulin concentrations were also higher in this group. However, even with this apparently minor increase of fasting glucose, impaired beta-cell function, assessed by both HOMA analysis and a raised proinsulin-to-C-peptide ratio, was seen in the hyperglycaemic siblings.

Both obesity and central distribution of obesity are known to be associated with insulin resistance and impaired glucose control. In our study, the hyperglycaemic siblings had a tendency to a higher BMI, though this did not reach statistical significance. On the other hand, they had a significant increase in waist circumference. This is probably not due to differences in precision in the two variables and supports the suggestion that centrality of fat distribution could be an earlier or more important feature of abnormal glucose metabolism than obesity in itself [26]. In spite of their increased insulin resistance and waist circumference, the sibling pairs did not differ significantly in blood pressure, triglycerides and cholesterol subfractions including HDL cholesterol. This observation is in accord with the previous cross-sectional comparison between normoglycaemic and hyperglycaemic siblings of Type II diabetic patients by Shaw, where, though hyperglycaemia was associated with reduced insulin sensitivity, no other differences in other Metabolic Syndrome variables were found. The lack of association with the wider spectrum of Metabolic Syndrome variables could be due to either the relatively small difference in glycaemia between the sibling pairs; or to the avoidance of the confounding effects of family 
heterogeneity seen in cross-sectional studies; or to type 2 error arising from the relatively small size of the study.

Hyperglycaemia is associated with increased cardiovascular risk [1] and first-degree relatives of Type II diabetic subjects have been shown to have a greater prevalence of cardiovascular disease $[12,27]$. This could partly be explained by abnormalities in haemostasis. Mansfield has previously shown elevated PAI1 antigen concentrations in normoglycaemic first-degree relatives of Type II diabetic subjects compared with a control population [18]. Although this was associated with differences in BMI and Metabolic Syndrome variables (triglycerides, HDL cholesterol and insulin resistance), these did not fully account for the observed differences in PAI-1. In our study, the hyperglycaemic siblings had considerably higher circulating PAI-1 concentrations than their paired normoglycaemic siblings. Indeed, there was a clearer separation between the groups with respect to PAI-1 than for any other measured variable. In the combined groups, PAI-1 was correlated univariately with BMI, waist circumference, insulin sensitivity and proinsulin concentrations, as has been previously described $[8,15]$. Of these, only waist circumference was associated with PAI-1 antigen in a step-wise regression analysis, though neither this, nor the combined associations of these factors was sufficient to explain the difference in PAI-1 between the groups. In a recent analysis of the Framingham Offspring Study, PAI-1 was associated with the fasting insulin concentration, a good measure of insulin resistance in normoglycaemic subjects [28]. However, as in this study, an additional and independent increase in PAI-1 concentration was seen in glucose intolerant subjects. PAI-1 synthesis and secretion by hepatic and endothelial cells are stimulated by glucose in vitro [29], but in vivo this is hard to disentangle from confounders such as the plasma concentrations of insulin, triglycerides and insulin precursors. The association between the proinsulin and PAI-1 concentrations noted in this study has been described both epidemiologically and in vitro. However, in this study, though proinsulin was univariately correlated with PAI-1, this association was displaced by waist circumference in the stepwise regression model.

In this study, t-PA was correlated with PAI-1 and was higher in the hyperglycaemic than in the normoglycaemic siblings, though this did not reach statistical significance. The contrast between the clear separation of PAI-1 and the marginal separation of t-PA between the normoglycaemic and hyperglycaemic groups could be a function of the small size of the study, or be associated with differences in their relationships to underlying factors such as the Metabolic Syndrome cluster, including central obesity and insulin resistance. Other abnormalities in both the fibrinolytic and coagulation systems have been described by
Mansfield et al in patients with Type II diabetes and their first-degree relatives when compared with normal controls, including raised fibrinogen and Factor VII:C [17]. In contrast to this, we found no differences and no consistent trends between the sibling pairs in other haemostatic variables. Though the study could be under-powered to detect such differences, the contrast with previous observations could have been due to more marked degrees of hyperglycaemia examined in other studies, or to their cross-sectional design.

The limitations of this study relate primarily to its size. This was due to the difficulty of identifying sex and age-matching non-diabetic pairs of siblings of Type II diabetic patients, discordant for fasting glycaemia. Only 13 such pairs were obtainable from a total of 313 large sibships (consisting of at least three members). The decision to compare paired siblings, though reducing the large between family cross-sectional variance due to shared genetic and environmental background also raises the question of whether the NG siblings represented a distinct phenotype by virtue of having a diabetic sibling, ie to what extent they were themselves metabolically 'abnormal'. They were more obese and insulin resistant than unselected nondiabetic control subjects (data not shown). However, when they were compared with a sex, age, BMI and FPG-matched group without a first-degree family history of diabetes, no significant differences were found in the Metabolic Syndrome variables measured, although haemostatic variables were not available for these controls and the numbers were again small. Insulin sensitivity was assessed using HOMA, and not independently measured with either the hyperinsulinaemic clamp or by IVGTT. Measurement conditions for HOMA (fasting) relate more to insulin action on the liver while clamp conditions relate to muscle response (as hepatic glucose production is largely suppressed). However, HOMA assessment correlates well with that of the clamp $[30,31]$ and HOMA $\% \mathrm{~S}$ also correlates highly with $S_{\mathrm{i}}$ assessed by minimal modelling of the IVGTT [19].

In summary, we have studied differences between sex- and age-matched sibling pairs discordant for fasting glycaemia below the (WHO 1985) diabetic range, to minimise intra-family differences in environment and genetic background and to avoid potential stratification bias. We have shown the hyperglycaemic siblings were more insulin resistant, had evidence of beta-cell dysfunction, were more centrally obese and had considerably raised PAI-1 concentrations. It is possible that the latter could contribute to cardiovascular risk in these subjects.

Acknowledgements. Professor R. Turner, who died suddenly in 1999, was central in the planning of this study. We are grateful to M. Stickland of the Unit of Molecular Vascular Medicine, the University of Leeds and the technical staff Diabetes Research Biochemistry Laboratory in the Nuffield Department of Medicine, University of Oxford for biochemical assays. 


\section{References}

1. Fuller JH, Shipley MJ, Rose G, Jarrett RJ, Keen H (1983) Mortality from coronary heart disease and stroke in relation to degree of glycaemia: the Whitehall study. BMJ 287: $867-870$

2. Kannel WB, McGee DL (1979) Diabetes and cardiovascular disease. The Framingham study. JAMA 241: 20352038

3. Garcia MJ, McNamara PM, Gordon T, Kannell WB (1974) Morbidity and mortality in diabetics in the Framingham population. Sixteen year follow-up. Diabetes 23: 105-111

4. Thompson S, Kienast MJ, Pyke S, Haverkate F, Loo JV (1995) Haemostatic Factors and the Risk of Myocardial Infarction or Sudden Death in Patients with Angina Pectoris. New Engl J Med 332: 635-641

5. Meade T, Brozovic M, Chakrabarti R et al. (1986) Haemostatic Function and Ischemic Heart Disease: Principle Results of the North Park Heart Study. Lancet 6: 533-537

6. Hamsten A, Eriksson P (1995) Fibrinolysis and Atherosclerosis. Ballieres Clin Haematol 8: 345-359

7. Davies M, Thomas A (1984) Thrombosis and Acute Coronary-Artery Lesions in Sudden Cardiac Ischemic Death. New Engl J Med 310: 1137-1140

8. Juhan-Vague I (1996) Thrombogenic and Fibrinolytic Factors and Cardiovascular Risk in Non-Insulin-dependent Diabetes Mellitus. Ann Med 28: 371-380

9. Fuller J, Keen H, Jarrett R et al. (1979) Haemostatic Variables Associared with Diabetes and its Complications. BMJ 2: 964-966

10. Morise T, Takkeuchi Y, Kawano M, Koni I, Takeda R (1995) Increased Plasma Levels of Immunoreactive Endothelin and von Willebrand Factor in NIDDM Patients. Diabetes Care 18: 87-89

11. Stewart M, Humphriss D, Berrish T et al. (1995) Features of syndrome $\mathrm{x}$ in first-degree relatives of NIDDM patients. Diabetes Care 18: 1020-1022

12. Haffner SM, Stern MP, Hazuda HP, Mitchell BD, Patterson JK, Ferrannini E (1989) Parental history of diabetes is associated with increased cardiovascular risk factors. Arteriosclerosis 9: 928-933

13. Walker M (1995) Obesity, Insulin resistance, and its Links to Non-Insulin Dependent Diabetes Mellitus. Metabolism 44: $18-20$

14. Shaw J, Purdie D, Neil H, Levy J, Turner R (1999) The relative risk of hyperglycaemia, obesity and dyslipidaemia in the relatives of patients with Type II diabetes mellitus. Diabetologia 42: 24-27

15. Landin K, Stigendal L, Eriksson E et al. (1990) Abdominal Obesity Is Associated with an Impaired Fibrinolytic Activity and Elevated Plasminogen Activator Inhibitor-1. Metabolism 39: 1044-1048

16. Landin K, Tengborn L, Smith U (1990) Elevated fibrinogen and plasminogen activator inhibitors (PAI-1) in hypertension are related to metabolic risk factors for cardiovascular disease. JIntern Med 227: 273-278

17. Mansfield MW, Heywood DM, Grant PJ (1996) Circulating levels of factor VII, fibrinogen, and von Willebrand factor and features of insulin resistance in first-degree relatives of patients with NIDDM. Circulation 94: 2171-2176
18. Mansfield MW, Stickland MH, Grant PJ (1997) PAI-1 concentrations in first-degree relatives of patients with non-insulin-dependent diabetes: metabolic and genetic associations. Thromb Haemost 77: 357-361

19. Hermans MP, Levy J, Morris RJ, Turner RC (1999) Comparison of insulin sensitivity tests across a range of glucose tolerance from normal to diabetes. Diabetologia 42: 678-687

20. Hermans MP, Levy JC, Morris RJ, Turner RC (1999) Comparison of tests of beta-cell function across a range of glucose tolerance from normal to diabetes. Diabetes 48: 17791786

21. UKPDS Group (1994) UK Prospective Diabetes Study XI: Biochemical risk factors in type 2 diabetic patients at diagnosis compared with age-matched normal subjects. Diabet Med 11: 534-544

22. Clauss A (1957) Gerinnungsphysiologische scnellmethode zur bestimmung des fibrinogens. Acta Haematol 17: 237-246

23. Song YC, Sheng D, Taubenfeld SM, Matsueda GR (1994) A microtiter assay for factor XIII using fibrinogen and biotinylcadaverine as substrates. Anal Biochem 223: 88-92

24. Zavaroni I, Mazza S, Luchetti L et al. (1990) High Plasma Insulin and Triglyceride Concentrations and Blood Pressure in Offspring of People With Impaired Glucose Tolerance. Diabet Med 7: 494-498

25. Lillioja S, Mott DM, Zawadzki JK et al. (1987) In vivo insulin action is familial characteristic in nondiabetic Pima Indians. Diabetes 36: 1329-1335

26. Han TS, Feskens EJ, Lean ME, Seidell JC (1998) Associations of body composition with Type 2 diabetes mellitus. Diabet Med 15: 129-135

27. Krolewski A, Czyzyk A, Kopczynski J, Rywik S (1981) Prevelance of Diabetes Mellitus, Coronary Heart Disease and Hypertension in the Families of Insulin Depedent and Insulin Independent Diabetes. Diabetologia 21: 520-524

28. Meigs J, D'Agostino R, Wilson P, Cupples L, Nathan D, Singer D (1997) Risk Variable Clustering in the Insulin Resistance Syndrome. The Framingham Offspring Study. Diabetes 46: 1594-1600

29. Nordt TK, Klassen KJ, Schneider DJ, Sobel BE (1993) Augmentation of synthesis of plasminogen activator inhibitor type-1 in arterial endothelial cells by glucose and its implications for local fibrinolysis. Arterioscler Thromb 13: 1822-1828

30. Bonora E, Targher G, Alberiche M et al. (2000) Homeostasis model assessment closely mirrors the glucose clamp technique in the assessment of insulin sensitivity: studies in subjects with various degrees of glucose tolerance and insulin sensitivity. Diabetes Care 23: 57-63

31. Lansang MC, Williams GH, Carroll JS (2001) Correlation between the glucose clamp technique and the homeostasis model assessment in hypertension. Am J Hypertens 14: 51-53

32. Matthews DR, Hosker JP, Rudenski AS, Naylor BA, Treacher DF, Turner RC (1985) Homeostasis model assessment: insulin resistance and $\beta$-cell function from fasting plasma glucose and insulin concentrations in man. Diabetologia 28: 412-419

33. Levy JC, Matthews DR, Hermans MP (1998) Correct homeostasis model assessment (HOMA) evaluation uses the computer program. Diabetes Care 21: 2191-2192 